\title{
THE CONCEPTUALIZATION AND USES OF TECHNOLOGICAL METAWORLDS IN TRAVEL
}

\author{
T.S. Stumpf \\ Humboldt State University \\ Tyler.Stumpf@humboldt.edu
}

\author{
Christopher B. Califf \\ Western Washington University \\ Christopher.Califf@wwu.edu
}

\author{
Joshua J. Frye \\ Humboldt State University \\ Joshua.Frye@humboldt.edu
}

\begin{abstract}
Mobile technology is increasingly interwoven into everyday life practices during travel. This study offers a theoretical extension of previous work on metaworlds by using an interpretive methodology to investigate how individuals conceptualize and mobile devices as technological metaworlds in travel. The findings suggest that while physical and technological metaworlds may initially seem to share some core use characteristics, a deeper dive into the data indicates an important difference regarding the uses and impacts of technological metaworlds. Specifically, concepts emerged which consistently pointed towards some adverse psychological impacts regarding the cognitive dissonance of technological metaworlds in travel. How and why this occurs is discussed through the delineation of a core conceptual category and three associated sub-categories. Finally, implications for theoretical and practical work on the technology - self nexus in travel are presented.
\end{abstract}

\section{Introduction}

Today, humans travel with an ever-increasing array of mobile technology. The traveler therefore regularly interacts with both the physical and digital worlds that shape the contours of their experience. How the traveler engages with, copes with, and makes sense of these worlds aligns with a concept known as touristic metaspatiality [17]. Touristic metaspatiality "indicates the qualities of spaces which increase the control of tourists and the dominance of their cultures in relation to the host community" [18, p. 2]. Such metaworlds are conceptualized and used as social, behavioral, and psychological safe-havens which allow the traveler to regain the locus of control and recover from heavily localized and enculturated situations in which the existing knowledge and mental models of the traveler are challenged. In contexts where the cultural differences between traveler and the local community are particularly high, the traveler is made to confront the new and unfamiliar in ways that produce a "stress of learning" effect, which in turn induces a desire to retreat to metaworlds [18, p. 3].

To date, the metaworlds of travel have only been conceptualized and discussed in the physical sense. However, the recent and rapid proliferation of mobile technology necessitates a conceptual revisiting of the influence of technology on the metaworlds of travel. The aim of the current study is to expand the theoretical depth of research in this area by advancing a more holistic conceptualization of touristic metaspatiality through provision for technology as a form of metaworld. To this end, this study draws on concepts embedded in sociomateriality [25] and uses semi-structured interviews to elucidate grounded insights on the uses and impacts of technological metaworlds.

The rest of the paper is organized as follows. First, we introduce and discuss the concept of physical metaworlds in travel. Next, we lay the conceptual foundation for an inquiry into technological metaworlds. This is followed by a discussion of our method and the results of the grounded investigation. We conclude with a discussion of some implications for research and practice.

\section{Literature review}

\subsection{Mobility and metaworlds}

Today, humans travel with an ever-increasing array of mobility options. This torrent of human mobility has opened the floodgates for countless areas of inquiry relating to the economic, sociological, psychological, and technological implications for the traveler, local residents, and all the existing or emergent physical and technological spaces between them. As such, peeling back the layers of complexity of travel phenomena continues to be in the emergent stages [6]. One still underdeveloped area of theoretical inquiry into travel is the study of metaworlds. 
A non-trivial byproduct of human mobility is exposure to other people, communities, and cultures. Ideally, this will benefit both the traveler and the visited communities via the potential for cross-cultural exchange and learning. However, in reality quite the opposite can occur as travelers "become confused, tired, and disoriented" [17, p. 452] from processing the new and different. Stated differently, travelers who hope to experience other places and cultures in raw, unmoderated and challenging ways will face the psychological and physiological fatigue of heightened cognitive and emotional processing. While each traveler has her own threshold for intentionally placing herself outside of her comfort zone, a threshold will eventually and inevitably be reached whereby the traveler will feel compelled to retreat into a metaworld. Metaworlds are formally defined as "realities placed in time and space that differ from the dominant reality" $[18$, p. 2]. In these restricted bubbles of psychological safety, the locus of control can be taken back from the dominant culture of the local community in which the traveler has entered into. By regulating exposure to the dominant reality of the host culture, metaworlds allow travelers to manage the tensions that can stem from experiencing and processing the idiosyncrasies of often complicated and not immediately comprehensible cultural contexts [18]. For example, a Norwegian traveler on a trip through Thailand will experience and inundation of novel and often confusing multi-sensory inputs. In order to help cope with the physiological fatigue (positive or negative) which often stems from this process, the traveler will find restricted metaworlds to take refuge in, regain control, and process the experience.

In his study of India, Hottola [18] discusses five types of metaworlds. The first is private spaces reserved for travelers, such as hotel rooms. The second is semi-private spaces of restricted access, such as shared spaces in hotels and guesthouses including restaurants, lobbies, and/or garden areas that facilitate interactions among fellow travelers. The third category is public spaces of restricted access, such as touristic areas that require an entrance fee. Next, spaces of temporary Western domination including heavily Westernized areas such as touristic beaches and social gatherings where members of the traveling out-group comprise the majority. The final category is wilderness areas, where the culture of tourists can freely flourish "in the 'cultural vacuum' of nature" [18, p. 6].

\subsection{Technology and metaworlds}

The proliferation of mobile technology necessitates a conceptual revisit that includes provision for the virtual/technological worlds in which today's traveler may utilize. While Wilson and Richards [33] recognize the importance of examining the spatial context of touristic metaworlds, the present study commenced with the basic idea that such contexts could be understood as both physical and technological.

As Law and Urry [22, p. 397] discuss, much of social science is predicated on a Euclidean perspective which posits "social worlds composed of discrete entities standing in hierarchical or inclusive relations with one another." Theories based on this perspective, such as structuration theory [12], assume spaces, places, people, and the associated experiences therein are temporally specific and dependent on physical proximity. In other words, to be present in a social setting (such as a metaworld), requires face-to-face interaction within a defined spatial setting characterized by physical immediacy. If these requirements are not met, then a social actor (such as a traveler) is thought to be absent from that social setting, thereby making presence and absence distinct states of being [12].

However, the advent of internet-based technology has given rise to other lines of thinking that blur the distinctions of temporal and spatial specificity. Even in structuration theory, there is provision for the idea that with modern technology and communication mediums, physical presence and proximity are not necessarily required for a social setting or experience to take place. Thus, technology represents a mechanism for "time space distanciation" which can overcome the constraints of time and space placed on social integration, and thus foster an individual's ability to transcend proxemics and normative temporal flows in their quest to fulfill social and even physiological needs [2], [12]. These ideas are also discussed through the concept of "extensibility" [1, p. 267], or an individual's ability to "overcome the friction of distance" through advances in technology. In this sense, the accessibility of technology, rather than proximity, becomes the catalyst for social integration, which in turn affects everything from the nature of work, familial exchanges, and even entire industries [1], such as travel and tourism.

Technology then allows for new possibilities for experiencing spaces and places by altering the primacy and exclusiveness of physical geographic location [16], [29]. This may constitute expanded ideas about the spaces and/or places that can be used as psychological safe-havens in travel, or other aspects of the metaworlds relied upon to enhance a traveler's sense of control and ability to moderate adaptation to new sociocultural contexts. Insomuch as technology is understood as those tools which allow a species to 
better control and adapt to its environment [26], it would seem that any discussion of the physical metaworlds utilized by travelers could be augmented by provision for the technological metaworlds accessible through mobile devices and applications.

\section{Method}

Classic grounded theory method (GTM) [13] was used in this research to develop provisional theoretical insights on if/how mobile technology factors into the metaworlds of travel. GTM was selected over other potentially useful interpretive methodologies (namely hermeneutic phenomenology) on account of its utility for systematically generating novel conceptual insights from data. Systematic theory generation was considered particularly important in this study, in that no theories on the technology - metaworld nexus have been hitherto delineated in the literature.

A core tenet of GTM research is theoretical sensitivity, which stems from a researcher's aptitude at integrating concepts generated in the data collection and analysis with existing theory [13]. As Stumpf and Califf [31] point out, there is a prevalent misconception about theoretical sensitivity in GTM which assumes that existing theory can be integrated in the theory development process only after the data has been collected and analyzed. While GTM researchers must not begin a project with preconceived ideas about what theoretical insights will emerge from the data collection and analysis, this is not to say that existing theoretical frameworks cannot be used to give focus to the research prior to the data collection and analysis. In fact, the use of sensitizing theory that provide initial parameters to the study can be considered a vital part of grounded research [30], [31].

In management information systems literature, Sarker et al. [30] refer to the application of a guiding meta-theoretical lens to grounded investigations as the principle of theoretical engagement. While it is typically assumed that qualitative researchers seeking to apply theory to their work must either do so initially, iteratively, or think of theory as the outcome [8], Sarker et al. [30] argue that this principle of theoretical engagement can help effectively use theory across all three of these phases. In other words, researchers can become sensitized to extant theory initially to provide focus to the data collection and analysis, which will facilitate the use and development of theoretical insights iteratively, thereby enhancing the probability of endemic theory development as the study's eventual outcome.

\subsection{Sociomateriality as guiding meta-theory}

Sociomateriality was used as the guiding metatheory in the present study to provide a general framework for the issues to be pursued in the data collection and analysis. Sociomateriality met all the tenets of an initial guiding meta-theory in that it was deemed as having a useful framing capacity, it was deemed reputable while also having good analytical dexterity as the jumping off point for the generation of novel insights, and was deemed as having high potential for knowledge production on the area of investigation [31].

As Orlikowski [25] states, many studies on technology adoption take either a techno-centric or human-centered perspective. The techno-centric perspective takes a functional approach to exploring how technology influences human actions, while the human-centered perspective attempts to understand how individuals "make sense of and interact with technology in various circumstances" [25, p. 1437]. Whereas the techno-centric perspective is criticized as ignoring sociocultural influences in technology adoption, the human-centered perspective is thought to downplay the technology itself, and over-emphasize sociocultural influences. In light of these respective shortcomings, Orlikowski [25, p. 1437] articulates the idea of sociomateriality, whereby both the social and material aspects of life are indistinguishably linked through "constitutive entanglement". Stated differently, sociomateriality assumes that humans and technology are intertwined to such an extent that neither is a distinct entity that can be viewed as having either one-way or two-way interactions on the other, but rather are entangled with the language, places, social interactions, and spatial arrangements of everyday life to an extent that humans and technology become conceptually, practically, and ontologically inextricable. In the initial data collection process, sociomateriality was merely used as a conceptual guide in order to frame preliminary questions on if/how/when technology is entangled with the self during travel, and what the impacts of this constitutive entanglement may be.

\subsection{Data collection and analysis}

The data collection was centered on conducting semi-structured interviews with subjects who had a recent travel experience in which they utilized mobile technology (i.e., smartphone). A total of 12 interviews were conducted, which occurred over a period of 23 days. Eight of the interviews were conducted with subjects whose participation had been pre-arranged, and four of the interviews were based on the theoretical 
sampling process [13]. These interviews ranged from 11 to 42 minutes $(\bar{X}=18)$. Of the 12 subjects, five used a recent international travel experience as the frame of reference for their responses, and seven used a recent domestic travel experience. All interviews were transcribed verbatim immediately upon completion by the first author of this study.

\subsubsection{Open coding}

Subsequent to transcription, open coding [13] was used to develop an initial understanding about the data by assigning conceptual codes to individual data incidents which were themselves comprised of 1-2 sentences of text. A total of 341 data incidents were examined. Constant comparison [13] was used throughout the analysis to compare open codes as they developed, and iteratively refine the codes as the emerging thematic concepts became clearer. Certain codes began to be consolidated together at a higher level of conceptual abstraction as the open coding progressed. This resulted in the development of a total of 49 distinct codes in the open coding phase.

During interview \#5, a data incident was open coded as "Phone creates cognitive dissonance during started to occur more frequently in the data, and were seemingly connected in ways which indicated the existence of various conceptual sub-categories of which cognitive dissonance was centrally positioned. In that the idea of integrating cognitive dissonance seemed to have relevant theoretical implications while also being highly variable conceptually, this concept met all the main criteria for a core conceptual category in classic GTM research [13]. As such, Cognitive Dissonance of Technological Metaworlds was selected as the core conceptual category, thereby helping to circumscribe the selective coding process, discussed next.

\subsubsection{Selective coding}

With the core category developed, the subsequent data collection and analyses transitioned to selective coding. Specifically, this stage of coding delimited subsequent lines of interview questions around select concepts deemed relevant to the evolving conceptual framework, used theoretical sampling to direct additional sources of data collection, and iteratively elevated certain groups of concepts into a higher level of abstraction through constant comparison [13].

Specifically, questions in the selective coding phase

Table 1. The coding and analysis process: Data incident to core conceptual category

\begin{tabular}{llll}
\hline Data incident & $\begin{array}{l}\text { Open coding } \rightarrow \\
\text { Selective coding }\end{array}$ & Sub-category & $\begin{array}{l}\text { Core } \\
\text { category }\end{array}$
\end{tabular}

I relied heavily on the phone for looking up Utilitarian use of information or other immediate needs. But technology in travel the phone is a distraction during travel, so I also wanted that time to unplug.
Must disconnect to truly experience
Cognitive Dissonance

of Technology as Tool

Phone as safe haven in

unfamiliar situations used my phone to talk to people back home and combat my depression. When I'm traveling I want to get a different perspective on life, and when I would connect too much with people back home it robs me of that new perspective.

My phone is like a universe on a screen. But I don't want to go somewhere just to do the thing I can do in my bedroom, which is mindlessly scrolling.
Phone as infinite space

Phone as mindless stimulation
Cognitive Dissonance of Technology as Safe Haven

travel experience

Phone as portal to 
entanglement during travel. Themes were increasingly grouped together in overarching conceptual subcategories relevant to the core category of Cognitive Dissonance of Technological Metaworlds. These subcategories were named: Cognitive Dissonance of Technology as Tool, Cognitive Dissonance of Technology as Safe Haven, and Cognitive Dissonance of Technology as Placeless Space. Table 1 presents an illustration of the open and selecting coding process of this research.

\subsubsection{Theoretical coding}

Subsequent to open and selective coding, theoretical coding was used to relate the codes conceptually as part of an organized theoretical framework. Theoretical coding is a more implicit process where "one talks substantively and thinks theoretically of the relationship between codes" [13, p. 72]. A key aspect to this is integrating relevant extant literature to conceptually elaborate the grounded insights being developed through theoretical sensitivity. Theoretical sensitivity helps raise the theory to higher levels until a final form can be reached.

The theoretical coding of the present study was centered on the Cognitive Dissonance of Technological Metaworlds and its three supporting sub-categories. Various streams of extant literature were drawn upon to aid conceptual elaboration using constant comparison, whereby the conceptual categories and extant knowledge were continuously compared with each other to generate insights throughout the theoretical coding process. The resultant grounded theory is presented next.

\section{Findings and discussion}

As Stumpf and Califf [31] explain, the initial and iterative use of a guiding meta-theory in GTM research requires a careful balance whereby the researcher must use discipline and discretion to ensure that the metatheory provides focus to the data collection and analysis without restricting serendipitous discovery in the generation of novel theoretical insights. The use of sociomateriality in the present study proved to be very useful for providing conceptual focus in the initial findings. The idea of constitutive entanglement [25] proved especially relevant to framing how mobile technology is integrated into travel experiences. For example, as opposed to treating technology as a kind of independent variable [26], the data quickly supported the idea that mobile technology is unconsciously entangled in the lived experience of the modern traveler. Initial codes such as "phone as psychological muscle memory", "unconscious use of technology", and "technology as an extension of self" supported this idea. As one subject explained the intertwinement of technology and self during travel:

When I don't have my phone it's like a piece of me is gone. It has almost become a part of my programmed identity.

This and similar comments made it clear that mobile devices are increasingly interwoven with everyday life practices during travel, and thus helped to efficiently direct the subsequent analysis toward developing a deeper understanding of this constitutive entanglement. The findings of this deep dive extend knowledge on how travelers use technology as a metaworld in travel and the associated psychological impacts, discussed next.

\subsection{Core category: Cognitive dissonance of technological metaworlds}

In his seminal work on the topic, Hottola [18, p. 2] describes metaworlds in travel as "bubbles" which provide an individual the ability to gain physical, behavioral, and/or psychological respite from situations and events in the external environment. Missing from this previous work on such metaworlds is provision for the potential of technology to serve as a kind of behavioral and/or psychological metaworld given the proliferation and use of mobile devices. Less intuitive are the nuances of technological metaspatiality uncovered through this research, especially when viewed through the prism of sociomateriality [25].

Specifically, the core conceptual category of the findings regarding cognitive dissonance suggest the seemingly adverse psychological impacts of technological metaworlds during travel and the constitutive entanglement of the self and technology. Cognitive dissonance stems from situations in which two or more items of information do not fit well with each other in the mind of an individual, and thereby incite behavioral and/or psychological processes that attempt to reconcile such belief, attitude, and/or behavior inconsistencies [10]. In the present study, cognitive dissonance factored into each of the conceptual sub-categories which relate to how and when information stemming from mobile technology created dissonance with information from the external environment during travel. 


\subsubsection{Sub-category 1: Cognitive dissonance of technology as tool}

A consistent theme to emerge from the data was that during the portions of travel characterized by high levels of vitality, where the traveler is immersed in navigating the fun and challenging aspects of the experience, and where there is a desire to more deeply engage with the commercial, social, and/or natural aspects of their external environment, mobile technology is frequently conceptualized and used as more of a tool. While mobile devices take on a high utility function for helping one manage and navigate a situation as with physical metaworlds [18], the escape function one typically associates with metaworlds is not present under the aforementioned conditions. As one subject stated:

I used [my phone] mostly as a tool to enhance what I was doing on the trip. I kind of saw it as a resource to look up information and knowledge for getting around and being able to experience another culture. Sometimes I would see something, look it up, and read about the history and the meaning of it to make a better connection with the place.

However, while statements like this provided evidence to support the idea that mobile technology supports a traveler's learning and understanding of a place via its utility function, the same traveler will also simultaneously experience an internal psychological struggle with their perceived need to either reduce or all-together abandon their mobile device to enhance their experience. Festinger [9] refers to these two competing and inconsistent cognitions as having a dissonant relationship, thereby producing cognitive dissonance. One subject explained this contradiction between the desire to use technology as a tool and the desire to limit/abandon technology in order to make a better connection with the place as follows:

At the same time, I want my trips to be an escape where I don't have to be on my phone because it is a distraction. When I'm traveling I want to get a different perspective on life, and when I arrive at a new destination I don't want to experience the destination through my phone. I want to just take it whatever I am doing or looking at.

The level of dissonance produced by this psychological struggle between two competing thoughts depends on the importance of the cognition to the traveler [9], or in this case how much value a traveler places on either utilizing technology as a tool to make a better connection to a place, or reducing technology to achieve the same end. The following proposition was developed from the ideas in this conceptual sub-category as part of the theoretical coding process.

Proposition 1: The level of a traveler's cognitive dissonance regarding technological metaworlds will depend on the importance of cognition gap between the value placed on using mobile technology as a tool to enhance the experience through better connection to a place and the value placed on reducing the use of mobile technology to enhance the experience through better connection to place.

\subsubsection{Sub-category 2: Cognitive dissonance of technology as safe haven}

Another strong theme to emerge from the data related to how and when mobile technology is used to actively disengage with the external environment during travel. The use and conceptualization of technology as a psychological safe haven aligns closely with Hottola's [18] original research on physical metaworlds, but with a caveat which seemingly pertains specifically to technological metaworlds. As with physical metaworlds [18], the findings of the present study indicate that in travel situations where factors in the external environment produce a stress of learning effect stemming from processing and adapting to the unfamiliar, an individual will seek psychological refuge in the technological metaworld. Throughout the course of the data collection, subjects repeatedly articulated how their mobile devices served as a psychological safe haven when faced with uncomfortable situations from which they wanted to disengage. As one subject explained:

When I was fed up at different times during the trip, I would find myself going into my phone to escape what was happening. It felt almost like a safe space. It was something familiar. I didn't have to engage with people I didn't know because I could go into my own little bubble in my phone.

As can be seen, the rhetoric used to describe technological metaworlds (i.e., escape, safe space, familiarity, bubble) align almost verbatim with those used to describe physical metaworlds [18]. However, two important factors seem to characterize technological metaworlds which are not discussed in physical metaworlds: unconscious reliance and cognitive dissonance. First, as opposed to the more conscious effort involved in entering physical metaworlds as discussed by Hottola [18], the data here indicate that entering technological metaworlds is done 
less deliberately and more unconsciously. As one subject stated:

I had nothing in common with the people, and so I would be on my phone the entire time. I didn't even really realize I was using it until people called me out on it, and asked me to put away my phone because they were trying to talk to me. Even when I was walking around I didn't even realize I was pulling it out and using it most of the time.

This aligns with the notion of constitutive entanglement in sociomateriality and the inextricable intertwinement of self and technology [25], whereby the boundary between the two are not always clear [11]. These ideas also appear in the literature on posthumanism, which postulates an evolutionary trajectory whereby humans and technology are increasingly merged such that individuals exists increasingly less in the physical sense and increasingly more in the virtual sense as a pattern of information [20], [24], or where the boundaries between self and technology are unclear or indistinguishable [3]. In other words, the biological conditionality of humankind is increasingly transcended as technology develops and becomes ever more integrated into our lives [21].

The present findings indicate that a key, although less recognized, aspect of this unconscious reliance on mobile technology as a psychological safe haven in travel is the cognitive dissonance seemingly associated with it. So strong is this association, based on these findings, that there indeed appears to be a causal relationship between unconscious reliance on technology as a safe haven and cognitive dissonance. Specifically, subjects consistently expressed the idea that even while utilizing technology as a psychological safe haven, they simultaneously harbored negative feelings toward such use and reliance. As such, these findings regarding the cognitive dissonance of technological metaworlds as a safe haven from the external environment represent a departure from the same purpose served by physical metaworlds as discussed by Hottola [18]. As one subject stated:

After, or sometimes even while I was looking at my phone, I would rarely think that was a valuable use of my time. It was just an automatic response to not wanting to deal with what was happening around me. I know I have to stop doing that.

As a result of these kinds of comments, the following propositions were developed as part of the theoretical coding process regarding technology as a safe haven and cognitive dissonance.
Proposition 2: There is significantly more cognitive dissonance associated with technological metaworlds as a psychological safe haven than with physical metaworlds.

Proposition 3: There is a direct and positive relationship between unconscious reliance on mobile technology as a psychological safe haven during travel and cognitive dissonance.

Proposition 4: The level of a traveler's cognitive dissonance regarding technological metaworlds will depend on the importance of cognition gap between the value placed on using mobile technology as a psychological safe haven from the external environment and the value placed on reducing the use of mobile technology as a psychological safe haven from the external environment.

\subsubsection{Sub-category 3: Cognitive dissonance of technology as placeless space}

Understandings of place and space are socially constructed and have thus evolved over time [4]. A long line of research across disciplines spanning human geography [15], [32] to philosophy [4], [7] to information systems [29] have recognized distinctions between place and space. Previous work on metaworlds does not include provision for such distinctions. For instance, Hottola [18] describes physical metaworlds as "spaces which increase the control of tourists" (p. 2), "restricted spaces that are used as places of recovery" (p. 2), "spatial realms" (p. 3), "self-made temporal niches" (p. 3), "places where [Western travelers] can isolate themselves" (p. 4), and "places of high degree of touristic metaspatiality" (p. 10). Indeed, the distinction between places and spaces is not always clear, and it is difficult to disentangle one from the other as they are dialectically structured together into the human experience [28], [32].

However, Sarker and Sahay [29, p. 4] point out that while little distinction is often made between the spaces and places of technology and information systems, making this distinction is important as these terms "reflect distinctive meanings and identifications of people to locations." Spaces are often described as locations that have not yet been imbued with meaning by an individual. Places, on the other hand, are described as locations of individual significance that have already been imbued with psychological, physiological, social and/or experiential meaning [32]. As Tuan [32, p. 3] states, "place is security, space is freedom: we are attached to the one and long for the other." Yet, conceptualizations of physical space and place are made more multi-dimensional by technology 
and the potential for virtual spaces/places therein. In the field of human geography, Haegerstrand [15] argued that space should be understood as a social rather than a physical construct. Given the myriad applications that connect the user elsewhere (i.e., social media, email, text messaging, etc.), mobile technology allows today's traveler to be decentered, fragmented, and dispersed across time and space.

As such, in an effort to provide greater conceptual clarity to these issues, questions relating to subjects' perceptions on the spaces or places of mobile technology in travel were integrated as the study progressed. Interestingly, and without any additional information or formal definitions of spaces and places from the principal investigator, the subjects of this research consistently responded that they viewed mobile devices (and all of the assorted apps and functions therein) as spaces, not places. Previous work has posited technology and digital networks as a kind of "placeless space" [5] that characterizes an increasingly networked society, and indeed may thought to be "layered on top of, within and between the fabric of traditional geographical space" [2, p. 616]. One perspective posits technology as a kind of liberating force that makes anything possible anywhere, given the ability to almost instantly access of world of information thereby creating the potential for spaceless cities [27] and a global village [23]. However, the results of this research suggest that greater provision is needed for potential consequences associated with the entanglement of this access to the placeless spaces of technological metaworlds and the geography of places during travel. As Graham [14, p. 167] states:

In the simple, binary allegations that new technologies help us to access a new 'electronic space' or 'place', which somehow parallels the lived material space of human territoriality, little conscious thought is put to thinking conceptually about how new information technologies actually relate to the space and places bound up with human territorial life.

A consistent and clear message across subjects of this research related to the adverse psychological impacts produced by the constitutive entanglement of technology and self during travel, and the constant pull that often unconsciously draws one into the placeless space of technology. This psychological impact frequently took the form of cognitive dissonance regarding the perceived usefulness of entering technological metaworlds immediately prior to doing so, and the perceived meaninglessness of the technological metaworld during and immediately after use. As one subject stated:
I used my phone to relax as kind of a habit. Then pretty soon I realized I was neglecting my own experience by using it to pretend to be someplace else, but I wasn't really there and I couldn't get a real personal connection and feeling. So my phone was like an unlimited space that limited my ability to experience the actual place I was in.

Another subject commented:

The phone was like muscle memory for my mind, an automatic response to boredom. But it was just mindless consumption that took me away from enjoying what I had in the moment. And you are supposed to be enjoying what you are doing when you travel.

Still another subject put it this way:

The phone takes me out of the place during travel. It's a space which has no meaning for me anymore. You are not fully experiencing what the place has to offer and are choosing to substitute with this mindless space that is totally infinite and can satisfy cravings, but can't give you real fun and entertainment. We let our imaginations leave us when it comes to experiencing actual places when you use technology too much.

These findings contrast the original ideas on the benefits and use of physical metaworlds in travel [18], and therefore help provide a theoretical extension toward understanding metaworlds more holistically. Whereas physical metaworlds are posited as offering the traveler greater control in the "fulfillment of personal goals and motives" by providing places of recovery, reflection, and learning [18, p. 2], it seems that technological metaworlds promise similar ends but in fact deliver a seemingly contrary result. The following propositions were developed as part of the theoretical coding process regarding the cognitive dissonance associated with the placeless spaces of technological metaworlds in travel.

Proposition 5: The perceived usefulness of entering technological metaworlds immediately prior to doing so will be less than the perceived meaninglessness of the technological metaworld immediately after.

Proposition 6: Technological metaworlds offer travelers significantly less opportunity for recovery, reflection, and learning than physical metaworlds.

\section{Conclusion}

In an increasingly technological world, it is important to understand that while technology is a social 
construction, societies and the people in them are a technological construction through technology's ability to shape human behavior in new ways [19]. This study examined how travelers use mobile technology by using GTM in conjunction with sociomateriality as a guiding meta-theory to frame the initial data collection and analysis. The findings suggest that while physical and technological metaworlds share some similarities in that they are both conceptualized and used as "bubbles" where the traveler can process information and/or use as a psychological retreat from the external environment, technological metaworlds seem to engender forms of cognitive dissonance which physical metaworlds do not. This is the first study of its kind on technological metaworlds, and thus provides travel, technology, psychology, sociology, and/or information systems researchers with a theoretical foothold from which to launch future studies on the impacts of mobile technology in travel experiences. Given that travel and technology are two of the biggest industries in the world, it is hoped that future research can further flesh out additional insights into the nexus between the two in order to better understand the impacts of the constitutive entanglement [25] of technology and the self.

\section{References}

[1] P. C. Adams, "A Reconsideration of Personal Boundaries in Space-Time," Annals of the Association of American Geographers, vol. 85, no. 2, 1995, pp. 267-285.

[2] D. Boden and H. L. Molotch, "The Compulsions of Proximity" In NowHere: Space, Time and Modernity (R. Friedland and D. Boden, Eds), pp. 257-286, University of California Press, Berkeley, 1994.

[3] R. Braidotti, The Posthuman, Polity, Cambridge, 2013.

[4] E. S. Casey, The Fate of Place: A Philosophical History, University of California Press, Berkeley, 1997.

[5] M. Castells, The Informational City: Information Technology, Economic Restructuring, and the Urban Regional Process, Blackwell Publishers, Oxford, 1989.

[6] T. Coles, C. M Hall, and D. T Duval, "Tourism and PostDisciplinary Enquiry," Current Issues in Tourism, vol. 9, no. 4-5, 2006, pp. 293-319.

[7] M. de Certeau, The Practice of Everyday Life, Berkeley, University of California Press, 1980.

[8] K. M. Eisenhardt, "Building Theories from Case Study Research," Academy of management Review, vol. 1, no. 4, 1989, pp. 532-550.
[9] L. Festinger, A Theory of Cognitive Dissonance, Row \& Peterson, Evanston, 1957.

[10] L. Festinger, "Cognitive Dissonance," Scientific American, vol. 207, no. 4, 1962, pp. 93-106.

[11] N. Gane, "Radical Post-Humanism: Friedrich Kittler and the Primacy of Technology," Theory, Culture \& Society, vol. 22 , no. 3, 2005, pp. 25-41.

[12] A. Giddens, The Constitution of Society: Outline of the Theory of Structuration, Polity Press, Cambridge, 1984.

[13] B. G. Glaser, Theoretical Sensitivity: Advances in Methodology of Grounded Theory, Sociological Press, Mill Valley, 1978.

[14] S. Graham, "The End of Geography or the Explosion of Space? Conceptualizing Space, Place and Information Technology," Progress in Human Geography, vol. 22, no. 2, 1998, pp. 165-185.

[15] S. Haegerstrand, Innovation Diffusion as a Spatial Process, Chicago Press, Chicago, 1967.

[16] C. Hine, Virtual Ethnography, Thousand Oaks, Sage Publications, 2000.

[17] P. Hottola, "Cultural Confusion: Intercultural Adaptation in Tourism," Annals of Tourism Research, vol. 31, no. 2, 2004, pp. 447-466.

[18] P. Hottola, "The Metaspatialities of Control Management in Tourism: Backpacking In India," Tourism Geographies, vol. 7, no. 1, 2005, pp. 1-22.

[19] S. Kirsch, "The Incredible Shrinking World? Technology and the Production of Space," Environment and Planning D: Society and Space, vol. 13, 1995, pp. 529-555.

[20] O. Krueger, "Gnosis in Cyberspace? Body, Mind and Progress in Posthumanism," Journal of Evolution \& Technology, vol. 14, no. 2, 2005, pp. 77-89.

[21] R. Kurzweil, The Age of Spiritual Machines: When Computers Exceed Human Intelligence. Penguin, New York, 1999.

[22] J. Law, and J. Urry, "Enacting the Social," Economy and Society, vol. 33, no. 3, 2004, pp. 390-410.

[23] M. McLuhan, Understanding Media: The Extensions of Man, McGraw-Hill, New York, 1964.

[24] H. Moravec, Mind Children. The Future of Robot and Human Intelligence. Harvard University Press, Cambridge, 1988.

[25] W. J. Orlikowski, "Sociomaterial Practices: Exploring Technology at Work," Organization Studies, vol. 28, no. 9, 2007, pp. 1435-1448. 
[26] W. J., Orlikowski, and S. V. Scott, "Sociomateriality: Challenging the Separation of Technology, Work and Organization," The Academy of Management Annals, vol. 2, no. 1, 2008, pp. 433-474.

[27] M. Pawley, Architecture, Urbanism and the New Media, Mimeo, 1995.

[28] E. Relph, Place and Placelessness, Pion Limited, London, 1976.

[29] S. Sarker and S. Sahay, "Implications of Space and Time for Distributed Work: An Interpretive Study of USNorwegian Systems Development Teams," European Journal of Information Systems, vol. 13, no. 3, 2004, pp. 3-20.

[30] S. Sarker, X. Xiao, and T Beaulieu, "Qualitative Studies In Information Systems: A Critical Review and Some
Guiding Principles," Management Information Systems Quarterly, vol. 37, no. 4. 2013, pp. iii-xvii.

[31] T. S. Stumpf and C. Califf, (2018). "On the Use of Meta-Theory in Grounded Investigations: In Principle and Practice in Hospitality and Tourism Research". In R. Nunkoo (Ed.), Handbook of Research Methods in Tourism and Hospitality Management. Edward Elgar:

[32] Y. F. Tuan, Space and Place: The Perspective of Experience, University of Minnesota Press, Minneapolis, 1977.

[33] J. Wilson, and G. Richards, "Suspending Reality: An Exploration of Enclaves and the Backpacker Experience," Current Issues in Tourism, vol. 11, no. 2, 2008, pp. 187-202. 